\title{
A 4D counter-example showing that DWCness does not imply CWCness in $n$-D
}

\author{
Nicolas Boutry ${ }^{1}$, Rocio Gonzalez-Diaz ${ }^{2}$, Laurent Najman ${ }^{3}$, and Thierry \\ Géraud $^{1}$ \\ EPITA Research and Development Laboratory (LRDE), EPITA, France \\ Universidad de Sevilla, Sevilla, Spain \\ Université Paris-Est, LIGM, Équipe A3SI, ESIEE, France
}

\begin{abstract}
In this paper, we prove that the two flavors of well-composedness called Continuous Well-Composedness (shortly CWCness) and Digital Well-Composedness (shortly DWCness) are not equivalent in dimension 4 thanks to an example of a configuration of 8 tesseracts (4D cubes) sharing a common corner (vertex), which is DWC but not CWC. This result is surprising since we know that CWCness and DWCness are equivalent in $2 \mathrm{D}$ and $3 \mathrm{D}$. To prove this new result, local (and then relative) homology are used. This paper has been submitted to IWCIA.
\end{abstract}

Keywords: well-composed, topological manifolds, critical configurations, digital topology, local homology

\section{Introduction}

Digital Well-Composedness is a strong property in digital topology, because it implies the equivalence of $2 n$ and $\left(3^{n}-1\right)$ connectivities [3] in a set and in its complement. A well-known application of this flavour of well-composedness is the tree of shapes $[7,9]$. On the other side, CWC images are known as "counterparts" of n-dimensional manifolds in the sense that the boundary of their continuous analog does not have singularities (no "pinches"). The consequence is that some geometric differential operators can be directly computed on the discrete sets, which simplifies or makes specific algorithms faster $[12,13]$. These two flavours of well-composednesses, known to be equivalent in 2D and in 3D, are not equivalent in $4 \mathrm{D}$, and this is what we are going to prove in this paper. Section 2 recalls the material relative to discrete topology and local homology necessary to the proof detailed in Section 3. Section 4 concludes the paper.

\section{Discrete topology}

As usual in discrete topology, we will only work with digital sets, that is, finite subsets of $\mathbb{Z}^{n}$ or subsets $\mathcal{X}$ of $\mathbb{Z}^{n}$ whose complementary set $\mathbb{Z}^{n} \backslash \mathcal{X}$ is finite. 


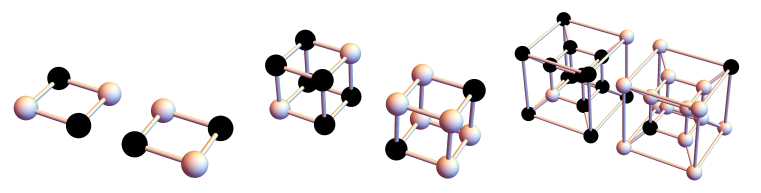

Fig. 1: Examples of primary and secondary 2D, 3D and 4D critical configurations. Black bullets correspond to points of a digital set $\mathcal{X}$ and white bullets correspond to points of $\mathcal{X}^{c}$.

\subsection{Digital well-composedness}

Let $n \geq 2$ be a (finite) integer called the dimension. Now, let $\mathbb{B}=\left\{e^{1}, \ldots, e^{n}\right\}$ be the (orthonormal) canonical basis of $\mathbb{Z}^{n}$. We use the notation $x_{i}$, where $i$ belongs to $\llbracket 1, n \rrbracket:=\{i \in \mathbb{Z} ; 1 \leq i \leq n\}$, to determine the $i^{\text {th }}$ coordinate of the vector $x \in \mathbb{Z}^{n}$. We recall that the $L^{1}$-norm of a point $x \in \mathbb{Z}^{n}$ is denoted by $\|\cdot\|_{1}$ and is equal to $\sum_{i \in \llbracket 1, n \rrbracket}\left|x_{i}\right|$ where $|\cdot|$ is the absolute value. Also, the $L^{\infty}$-norm is denoted by $\|\cdot\|_{\infty}$ and is equal to $\max _{i \in \llbracket 1, n \rrbracket}\left|x_{i}\right|$.

For a given point $x \in \mathbb{Z}^{n}$, the $2 n$-neighborhood in $\mathbb{Z}^{n}$, denoted by $\mathcal{N}_{2 n}(x)$, is equal to $\left\{y \in \mathbb{Z}^{n} ;\|x-y\|_{1} \leq 1\right\}$. Also, the $\left(3^{n}-1\right)$-neighborhood in $\mathbb{Z}^{n}$, denoted by $\mathcal{N}_{3^{n}-1}(x)$, is equal to $\left\{y \in \mathbb{Z}^{n} ;\|x-y\|_{\infty} \leq 1\right\}$. Let $\xi$ be a value in $\left\{2 n, 3^{n}-1\right\}$. The starred $\xi$-neighborhood of $x \in \mathbb{Z}^{n}$ is noted $\mathcal{N}_{\xi}^{*}(x)$ and is equal to $\mathcal{N}_{\xi}(x) \backslash\{x\}$. An element of the starred $\xi$-neighborhood of $x \in \mathbb{Z}^{n}$ is called a $\xi$-neighbor of $x$ in $\mathbb{Z}^{n}$. Two points $x, y \in \mathbb{Z}^{n}$ such that $x \in \mathcal{N}_{\xi}^{*}(y)$ or equivalently $y \in \mathcal{N}_{\xi}^{*}(x)$ are said to be $\xi$-adjacent. A finite sequence $\left(p^{0}, \ldots, p^{k}\right)$ of points in $\mathbb{Z}^{n}$ is a $\xi$-path if and only if $p^{0}$ is $\xi$-adjacent only to $p^{1}, p^{k}$ is $\xi$-adjacent only to $p^{k-1}$, and if for $i \in \llbracket 1, k-1 \rrbracket, p^{i}$ is $\xi$-adjacent only to $p^{i-1}$ and to $p^{i+1}$. A digital set $\mathcal{X} \subset \mathbb{Z}^{n}$ is said $\xi$-connected if for any pair of points $x, y \in \mathcal{X}$, there exists a $\xi$-path joining them into $\mathcal{X}$. A $\xi$-connected subset $C$ of $\mathcal{X}$ which is maximal in the inclusion sense, that is, there is no $\xi$-connected subset of $\mathcal{X}$ which is greater than $C$, is said to be a $\xi$-component of $\mathcal{X}$.

For any $z \in \mathbb{Z}^{n}$ and any $\mathcal{F}=\left(f^{1}, \ldots, f^{k}\right) \subseteq \mathbb{B}$, we denote by $S(z, \mathcal{F})$ the set:

$$
\left\{z+\sum_{i \in \llbracket 1, k \rrbracket} \lambda_{i} f^{i} ; \lambda_{i} \in\{0,1\}, \forall i \in \llbracket 1, k \rrbracket\right\} .
$$

We call this set the block associated with the pair $(z, \mathcal{F})$; its center is $z+\sum_{f \in \mathcal{F}} \frac{f}{2}$, and its dimension, denoted by $\operatorname{dim}(S)$, is equal to $k$. More generally, a set $S \subset \mathbb{Z}^{n}$ is said to be a block if there exists a pair $(z, \mathcal{F}) \in \mathbb{Z}^{n} \times \mathcal{P}(\mathbb{B})$ such that $S=$ $S(z, \mathcal{F})$. We say that two points $p, q \in \mathbb{Z}^{n}$ belonging to a block $S$ are antagonists in $S$ if the distance between them equals the maximal distance using the $L^{1}$ norm between two points in $S$; in this case we write $p=\operatorname{antag}_{S}(q)$. Note that the antagonist of a point $p$ in a block $S$ containing $p$ exists and is unique. Two points that are antagonists in a block of dimension $k \geq 0$ are said to be $k$-antagonists; $k$ is then called the order of antagonism between these two points. We say that a digital subset $\mathcal{X}$ of $\mathbb{Z}^{n}$ contains a critical configuration in a block $S$ of dimension 
$k \in \llbracket 2, n \rrbracket$ if there exists two points $\left\{p, p^{\prime}\right\} \in \mathbb{Z}^{n}$ that are antagonists in $S$ such that $\mathcal{X} \cap S=\left\{p, p^{\prime}\right\}$ (primary case) or such that $S \backslash \mathcal{X}=\left\{p, p^{\prime}\right\}$ (secondary case). Figure 1 depicts examples of critical configurations. Then, a digital set $\mathcal{X} \subset \mathbb{Z}^{n}$ is said to be digitally well-composed (DWC) [3] if it does not contain any critical configuration.

\subsection{Basics in topology and continuous well-composedness}

Let $X$ be a set of points, and let $\mathcal{U}$ be a set of subsets of $X$ such that:

- $X, \emptyset \in \mathcal{U}$,

- any union of any family of elements in $\mathcal{U}$ belongs to $\mathcal{U}$,

- any finite intersection of any family of elements in $\mathcal{U}$ belongs to $\mathcal{U}$. (TO3)

Then $\mathcal{U}$ is said to be a topology, and the couple $(X, \mathcal{U})$ is called a topological space $[11,1]$. The elements of $X$ are called the points of $(X, \mathcal{U})$, and the elements of $\mathcal{U}$ are called the open sets of $(X, \mathcal{U})$. We will abusively say that $X$ is a topological space, assuming it is supplied with its topology $\mathcal{U}$. An open set which contains a point of $X$ is said to be a neighborhood of this point. Let $(X, \mathcal{U})$ be a topological space, and let $T$ be a subset of $X$. A set $T \subseteq X$ is said closed if it is the complement of an open set in $X$. A function $f: X \rightarrow Y$ between two topological spaces $(X, \mathcal{U})$ and $(Y, \mathcal{V})$ is continuous if for every open set $V \subset Y$, the inverse image $\left.f^{-1}(V)=\{x \in X \mid f(x) \in V\}\right\}$ is an open subset of $X$. The function $f$ is a homeomorphism if it is bicontinuous and bijective. As defined in [16], a topological space $M$ is said to be locally Euclidian of dimension $n \geq 0$ at $x^{0} \in M$ if $x^{0}$ has a neighborhood that is homeomorphic to an open subset of $\mathbb{R}^{n}$. Also, a second countable space is a topological space $X$ whose topology has a countable basis, that is, there exists some countable collection $\mathcal{U}=\left\{U_{i}\right\}_{i=1}^{\infty}$ of open sets of $X$ such that any open subset of $X$ can be written as a union of elements of some subfamily of $\mathcal{U}$. A Hausdorff space is a topological space where distinct points have disjoint neighborhoods. As recalled in [16], an n-dimensional topological manifold $M$ with $n \geq 0$ is a second countable Hausdorff space that is locally Euclidian of dimension $n$ at each $x^{0} \in M$. The continuous analog $\mathrm{CA}(p)$ of a point $p \in \mathbb{Z}^{n}$ is the closed unit cube centered at this point with faces parallel to the coordinate planes $\mathrm{CA}(p)=\left\{q \in \mathbb{R}^{n} ;\|p-q\|_{\infty} \leq 1 / 2\right\}$. The continuous analog $\mathrm{CA}(\mathcal{X})$ of a digital set $\mathcal{X} \subset \mathbb{Z}^{n}$ is the union of the continuous analogs of the points belonging to the set $\mathcal{X}$, that is, $\mathrm{CA}(\mathcal{X})=\bigcup_{p \in \mathcal{X}} \mathrm{CA}(p)$. Then, we will denote $\operatorname{bdCA}(\mathcal{X})$ the topological boundary of $\mathrm{CA}(\mathcal{X})$, that is, $\operatorname{bdCA}(\mathcal{X})=\mathrm{CA}(\mathcal{X}) \backslash \operatorname{Int}(\mathrm{CA} \mathcal{X}))$, where $\operatorname{Int}(\mathrm{CA}(\mathcal{X}))$ is the union of all open subsets of $\operatorname{CA}(\mathcal{X})$. Let $\mathcal{X} \subset \mathbb{Z}^{n}$ be a digital set. We say that $\mathcal{X}$ is a continuously well-composed set $(C W C)[14,15]$ if the boundary of its continuous analog $\operatorname{bdCA}(\mathcal{X})$ is a $(n-1)$-manifold, that is, if for any point $p \in \mathcal{X}$, the (open) neighborhood of $p$ in $\operatorname{bdCA}(\mathcal{X})$ is homeomorphic to $\mathbb{R}^{n-1}$. This property is self-dual: for any digital set $\mathcal{X} \subset \mathbb{Z}^{n}$, $\operatorname{bdCA}(\mathcal{X})=\operatorname{bdCA}\left(\mathcal{X}^{c}\right)$ and then $\mathcal{X}$ is continuously well-composed iff $\mathcal{X}^{c}$ is continuously well-composed. 


\subsection{Homomorphisms}

Recalls about Abelian groups can be found in [10]. Let $G$ and $G^{\prime}$ be two Abelian groups. A map $f: G \rightarrow G^{\prime}$ is called a homomorphism if $f\left(m_{1} g_{1}+m_{2} g_{2}\right)=$ $m_{1} f\left(g_{1}\right)+m_{2} f\left(g_{2}\right)$ for any $m_{1}, m_{2} \in \mathbb{Z}$ and for any $g_{1}, g_{2} \in G$. Let $G$ and $G$ be two free Abelian groups, and let $f: G \rightarrow G^{\prime}$ be a homomorphism. The set im $f:=\left\{b \in G^{\prime}: \exists a \in G, f(a)=b\right\}$ is called the image of $f$ in $\left.B\right]$. The set $\operatorname{ker} f:=\{a \in G ; f(a)=0\}$ is called the kernel of $f$. Note that $\operatorname{im} f$ is a subgroup of $G^{\prime}$ and that ker $f$ is a subgroup of $G$. A homomorphism $f$ is called an isomorphism if it is bijective. Two free Abelian groups are said isomorphic if there exists an isomorphism between them; for $A$ and $B$ two free Abelian groups, we write $A \simeq B$ when $A$ and $B$ are isomorphic. Let $A$ be a free Abelian group and $B$ a sugbroup of $A$. For each $a \in A$, defined the equivalence class $[a]:=\{a+b ; b \in B\}$. The quotient group $A / B$ is defined as $A / B:=\{[a] ; a \in A\}$.

Theorem 1 (First Isomorphism Theorem [10]). Let $A$ and $B$ be two free Abelian groups and $f: A \rightarrow B$ a homomorphism. Then $A / \operatorname{ker} f \simeq \operatorname{im} f$.

\subsection{Cubical sets}

An elementary interval is a closed subinterval of $\mathbb{R}$ of the form $[l, l+1]$ or $\{l\}$ for some $l \in \mathbb{Z}$. Elementary intervals that consist of a single point are degenerate, while those of length 1 are non-degenerate. An elementary cube $h$ is a finite product of elementary intervals, that is, $h=h_{1} \times \cdots \times h_{d}=\times_{i \in \llbracket 1, d \rrbracket} h_{i} \subset$ $\mathbb{R}^{d}$ where each $h_{i}$ is an elementary interval. The set of elementary cubes in $\mathbb{R}^{d}$ is denoted by $\mathcal{K}^{d}$. The set of all elementary cubes is $\mathcal{K}:=\bigcup_{d=1}^{\infty} \mathcal{K}^{d}$. Let $h=\times_{i \in \llbracket 1, d \rrbracket} h_{i} \subset \mathbb{R}^{d}$ be an elementary cube. The elementary interval $h_{i}$ is referred to as the $i$ th component of $h$. The dimension of $h$ is defined to be the number of non-degenerate components in $h$ and is denoted by $\operatorname{dim}(h)$. Also, we define $\mathcal{K}_{k}:=\{h \in \mathcal{K} ; \operatorname{dim}(h)=k\}$ and $\mathcal{K}_{k}^{d}:=\mathcal{K}_{k} \cap \mathcal{K}^{d}$. A set $X \subset \mathbb{R}^{d}$ is cubical if $X$ can be written as a finite union of elementary cubes. If $X$ is a cubical set, we adopt the following notation $\mathcal{K}(X):=\{h \in \mathcal{K} ; h \subseteq X\}$ and $\mathcal{K}_{k}(X):=\{h \in \mathcal{K}(X) ; \operatorname{dim}(h)=k\}$.

\subsection{Homology}

Let $X \subseteq \mathbb{R}^{d}$ be a cubical set. The $k$-chains of $X$, denoted by $C_{k}(X)$, is the free Abelian group generated by $\mathcal{K}_{k}(X)$. The boundary homomorphism $\partial_{x}^{X}$ : $C_{k}(X) \rightarrow C_{k-1}(X)$ is defined on the elementary cubes of $\mathcal{K}_{k}(X)$ and extended to $C_{k}(X)$ by linearity (see [10]). The chain complex $\mathcal{C}(X)$ is the graded set $\left\{C_{k}(X), \partial_{k}^{X}\right\}_{k \in \mathbb{Z}}$. A $k$-chain $z \in C_{k}(X)$ is called a cycle in $X$ if $\partial_{k}^{X} z=0$. The set of all $k$-cycles in $\mathrm{X}$, which is denoted by $Z_{k}(X)$, is $\operatorname{ker} \partial_{k}^{X}$ and forms a subgroup of $C_{k}(X)$. A $k$-chain $z \in C_{k}(X)$ is called a boundary in $X$ if there exists $c \in C_{k+1}(X)$ such that $\partial_{k}^{X} c=z$. Thus the set of boundary elements in $C_{K}(X)$, which is denoted by $B_{k}(X)$, consists of the image of $\partial_{k+1}^{X}$. Since $\partial_{k+1}^{X}$ 
is a homomorphism, $B_{K}(X)$ is a subgroup of $C_{k}(X)$. Since $\partial_{k}^{X} \partial_{k+1}^{X}=0$, every boundary is a cycle and thus $B_{k}(X)$ is a subgroup of $Z_{k}(X)$. We say that two cycles $z_{1}, z_{2} \in Z_{k}(X)$ are homologous and write $z_{1} \sim z_{2}$ if $z_{1}-z_{2}$ is a boundary in $X$, that is, $z_{1}-z_{2} \in B_{k}(X)$. The equivalence classes are then the elements of the quotient group $Z_{k}(X) / B_{k}(Z)$. The $k$-th homology group of $X$ is the quotient group $\mathbb{H}_{k}(X):=Z_{k}(X) / B_{k}(X)$. The homology of $X$ is the collection of all homology groups of $X$. The shorthand notation for this is $\mathbb{H}(X):=\left\{H_{k}(X)\right\}_{k \in \mathbb{Z}}$. Given $z \in Z_{k}(X),[z]$ is the homology class of $z$ in $X$. A sequence of vertices $V_{0}, \ldots, V_{n} \in \mathcal{K}_{0}(X)$ is an edge path in $X$ if there exists edges $E_{1}, \ldots, E_{n} \in \mathcal{K}_{1}(X)$ such that $V_{i-1}, V_{i}$ are the two faces of $E_{i}$ for $i=1, \ldots, n$. For $V, V^{\prime} \in \mathcal{K}_{0}(X)$, we write $V \sim_{X} V^{\prime}$ if there exists an edge path $V_{0}, \ldots, V_{n} \in \mathcal{K}_{0}(X)$ in $X$ such that $V=V_{0}$ and $V^{\prime}=V_{n}$. We say that $X$ is edge-connected if $V \sim_{X} V^{\prime}$ for any $V, V^{\prime} \in \mathcal{K}_{0}(X)$. For $x \in X$ we define the edge-connected component of $x$ in $X$ as the union of all edge-connected cubical subsets of $X$ that contain $x$. We denote it $\operatorname{ecc}_{X}(x)$. Using cubical sets, edge-connectedness is equivalent to (topological) connectedness $^{1}$ as stated by Theorem 2.55 p. 68 of [10]. This way, edge-connected components of $X$ are connected components of $X$ and conversely.

Theorem 2 ([10]). Let $X$ be a cubical set. Then $\mathbb{H}_{0}(X)$ is a free Abelian group. Furthermore, if $\left\{P_{i} ; i \in \llbracket 1, n \rrbracket\right\}$ is a collection of vertices in $X$ consisting of one vertex from each component of $X$, then $\left\{\left[\widehat{P}_{i}\right] \in \mathbb{H}_{0}(X) ; i \in \llbracket 1, n \rrbracket\right\}$ forms a basis for $\mathbb{H}_{0}(X)$.

\subsection{Relative homology}

Now, we recall some background in matter of relative homology. A pair of cubical sets $X$ and $A$ with the property that $A \subseteq X$ is called cubical pair and is denoted by $(X, A)$. Relative homology is used to compute how two spaces $A, X$ such that $A \subseteq X$ differ from each other. Intuitively, we want to compute the homology of $X$ modulo $A$ : we want to ignore the set $A$ and everything connected to it. In other words, we want to work with chains belonging to $C(X) / C(A)$, which leads to the following definition:

Definition 3 (Def. 9.3 of [10]). Let $(X, A)$ be a cubical pair. The relative chains of $X$ modula $A$ are the elements of the quotient groups $C_{k}(X, A):=$ $C_{k}(X) / C_{k}(A)$. The equivalence class of a chain $c \in \mathcal{C}(X)$ relative to $\mathcal{C}(A)$ is denoted by $[c]_{A}$. Note that for each $k, C_{k}(X, A)$ is a free Abelian group. The relative chain complex of $X$ modulo $A$ is given by $\left\{C_{k}(X, A), \partial_{k}^{(X, A)}\right\}_{k \in \mathbb{Z}}$ where $\partial_{k}^{(X, A)}: C_{k}(X, A) \rightarrow C_{k-1}(X, A)$ is defined by $\partial_{k}^{(X, A)}[c]_{A}:=\left[\partial_{k}^{X} c\right]_{A}$. Obviously, this map satisfies that $\partial_{k}^{(X, A)} \partial_{k+1}^{(X, A)}=0$. The relative chain complex gives rise to the relative $k$-cycles: $Z_{k}(X, A):=\operatorname{ker} \partial_{k}^{(X, A)}$, the relative $k$ boundaries $B_{k}(X, A):=\operatorname{im} \partial_{k+1}^{(X, A)}$, and finally the relative homology groups: $\mathbb{H}_{k}(X, A):=Z_{k}(X, A) / B_{k}(X, A)$.

\footnotetext{
${ }^{1}$ A set $X$ is said connected if it is not the union of two disjoint open non-empty sets.
} 
Proposition 4 (Proposition 9.4 p. 281 of [10]). Let $X$ be an (edge-)connected cubical set and let $A$ be a nonempty cubical set of $X$. Then, $\mathbb{H}(X, A)=0$.

\subsection{Exact sequences}

A sequence of groups and homomorphisms $\cdots \rightarrow G_{3} \stackrel{\psi_{3}}{\longrightarrow} G_{2} \stackrel{\psi_{2}}{\longrightarrow} G_{1} \rightarrow \ldots$ is said exact at $G_{2}$ if im $\psi_{3}=\operatorname{ker} \psi_{2}$. It is an exact sequence if it is exact at every group. If the sequence has a first or a last element, then it is automatically exact at that group. A short exact sequence is an exact sequence of the form $0 \rightarrow G_{3} \stackrel{\psi_{3}}{\longrightarrow} G_{2} \stackrel{\psi_{2}}{\longrightarrow} G_{1} \rightarrow 0$. A long exact sequence is an exact sequence with more than three nonzero terms.

Example 5 (Ex. 9.21 of [10]). The short exact sequence of the pair $(X, A)$ is:

$$
0 \longrightarrow C_{k}(A) \stackrel{\iota_{k}}{\longrightarrow} C_{k}(X) \stackrel{\pi_{k}}{\longrightarrow} C_{k}(X, A) \longrightarrow 0
$$

where $\iota_{k}$ is the inclusion map and $\pi_{k}$ is the quotient map.

Definition 6 (Def. 9.24 of [10]). Let $\mathcal{A}=\left\{A_{k}, \partial_{k}^{A}\right\}_{k \in \mathbb{Z}}, \mathcal{B}=\left\{B_{k}, \partial_{k}^{B}\right\}_{k \in \mathbb{Z}}$, and $\mathcal{C}=\left\{C_{k}, \partial_{k}^{C}\right\}_{k \in \mathbb{Z}}$ be chain complexes. Let 0 denote the trivial chain complex. Let $\phi: \mathcal{A} \rightarrow \mathcal{B}$ and $\psi: \mathcal{B} \rightarrow \mathcal{C}$ be chain maps. The following is a a short exact sequence of chain complexes:

$$
0 \longrightarrow \mathcal{A} \stackrel{\phi}{\longrightarrow} \mathcal{B} \stackrel{\psi}{\longrightarrow} \mathcal{C} \longrightarrow 0
$$

if, for every $k$, the sequence below is short exact:

$$
0 \longrightarrow A_{k} \stackrel{\phi_{k}}{\longrightarrow} B_{k} \stackrel{\psi_{k}}{\longrightarrow} C_{k} \longrightarrow 0
$$

Theorem 7 (Zig-zag Lemma (Th. 9.25 of [10])). Let $0 \rightarrow \mathcal{A} \rightarrow \mathcal{B} \rightarrow \mathcal{C} \rightarrow 0$ be a short exact sequence of chain complexes. Then, for each $k$, there exists a homomorphism $\partial_{*}: \mathbb{H}_{k+1}(\mathcal{C}) \rightarrow \mathbb{H}_{k}(\mathcal{A})$ such that

$$
\cdots \rightarrow \mathbb{H}_{k+1}(\mathcal{A}) \stackrel{\phi_{*}}{\longrightarrow} \mathbb{H}_{k+1}(\mathcal{B}) \stackrel{\psi_{*}}{\longrightarrow} \mathbb{H}_{k+1}(\mathcal{C}) \stackrel{\partial_{*}}{\longrightarrow} \mathbb{H}_{k}(\mathcal{A}) \rightarrow \ldots
$$

is a long exact sequence.

Corollary 8 (Exact homology sequence of a pair [10])). Let $(X, A)$ be a cubical pair. Then there is a long exact sequence

$$
\cdots \rightarrow \mathbb{H}_{k+1}(A) \stackrel{\iota_{*}}{\longrightarrow} \mathbb{H}_{k+1}(X) \stackrel{\pi_{*}}{\longrightarrow} \mathbb{H}_{k+1}(X, A) \stackrel{\partial_{*}}{\longrightarrow} \mathbb{H}_{k}(A) \rightarrow \ldots
$$

where $\iota: \mathcal{C}(A) \hookrightarrow \mathcal{C}(X)$ is the inclusion map and $\pi: \mathcal{C}(X) \rightarrow \mathcal{C}(X, A)$ is the quotient map. 


\subsection{Manifolds and local homology}

A subset $X$ of $\mathbb{R}^{n}$ is said to be locally an $n$-dimensional homological manifold at $x^{0} \in X$ if the homology groups $\left.\left\{\mathbb{H}_{i}\left(X, X \backslash\left\{x^{0}\right\}\right)\right)\right\}_{i \in \mathbb{Z}}$ satisfy:

$$
\left.\mathbb{H}_{i}\left(X, X \backslash\left\{x^{0}\right\}\right)\right)= \begin{cases}\mathbb{Z} & \text { when } i=n, \\ 0 & \text { otherwise. }\end{cases}
$$

Then, $X$ is said to be a $n$-dimensional homology manifold if it is locally an $n$-dimensional homology manifold at each point $x^{0} \in X$.

Theorem 9 ([18]). A topological manifold is a homology manifold.

\subsection{Homotopical equivalence}

Let $X, Y$ be two topological spaces, and $f, g$ be two continuous functions from $X$ to $Y$. We say that $f$ and $g$ are homotopic if there exists a continuous function $H: X \times[0,1] \rightarrow Y$ such that for any $x \in X, H(x, 0)=f(x)$ and $H(x, 1)=g(x)$. Furthermore, we say that $X$ and $Y$ are homotopically equivalent if there exist $f: X \rightarrow Y$ and $g: Y \rightarrow X$ such that $g \circ f$ is homotopic to $\operatorname{Id}_{x}$ and $f \circ g$ is homotopic to $\operatorname{Id}_{Y}$.

\section{DWCness does not imply CWCness}

It is well-known that DWCness and CWCness are equivalent in $2 \mathrm{D}$ and $3 \mathrm{D}$ (see, for example, [4]). In this section, we prove that there exists at least one set $\mathcal{X} \subset \mathbb{Z}^{4}$ which is DWC but not CWC. To this aim, we will start with the definition of $\mathcal{X}$ and we will observe that this set is DWC. Then, to prove that $\mathcal{X}$ is not $\mathrm{CWC}$, we will prove that $X=\operatorname{bdCA}(\mathcal{X})$ (up to a translation) is not a homology manifold and conclude that it is not a manifold by Theorem 9 . To compute the homology groups $\left\{\mathbb{H}_{i}\left(X, X \backslash\left\{x^{0}\right\}\right)\right\}_{i \in \mathbb{Z}}$, where $x^{0}$ is a particular point in $X$ (detailed hereafter), we need to compute $\left\{\mathbb{H}_{i}\left(X \backslash\left\{x^{0}\right\}\right)\right\}_{i \in \mathbb{Z}}$ and $\left\{\mathbb{H}_{i}(X)\right\}_{i \in \mathbb{Z}}$. However, $A:=X \backslash\left\{x^{0}\right\}$ is not a cubical set, then we need to find a cubical set $\widetilde{X}\left(x^{0}\right)$ which is homotopy equivalent to $A$ to compute its homology groups using the CHomP software package [8]. After having defined $\widetilde{X}\left(x^{0}\right)$ and having proven that it is a cubical set, we will show that $A$ and $\widetilde{X}\left(x^{0}\right)$ are homotopically equivalent. Then, we will compute the homology groups of $\widetilde{X}\left(x^{0}\right)$ and of $X$; this way we will deduce $\left\{\mathbb{H}_{i}\left(X, X \backslash\left\{x^{0}\right\}\right)\right\}_{i}$ using the long exact sequence of the pair $\left(X, X \backslash\left\{x^{0}\right\}\right)$. At this moment, we will see that $X$ is not a homology 3 -manifold, which will make us able to conclude that $\mathcal{X}$ is not CWC since the boundary of its continuous analog is not a topological 3-manifold. This way, we will conclude that DWCness does not imply CWCness in 4D. 


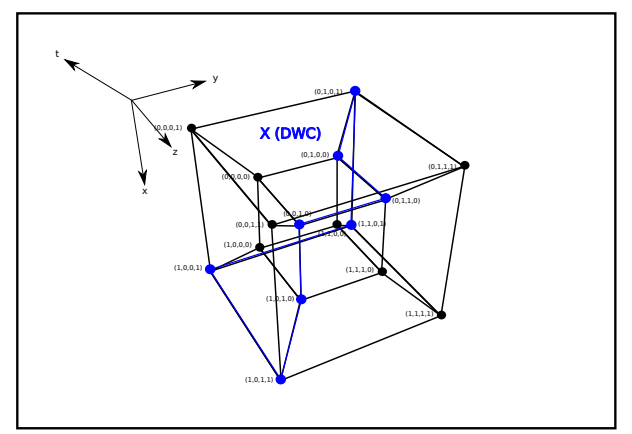

Fig. 2: A set $X_{0}$ depicted by blue points which is DWC and not CWC.

\subsection{Choosing a particular DWC set $\mathcal{X} \subset \mathbb{Z}^{4}$}

We recall that it is well-known in the community of discrete topology that CWCness and DWCness are equivalent in $2 \mathrm{D}$ and $3 \mathrm{D}$ as developed in $[2,4]$. For this reason, we chose a digital set $\mathcal{X}$ in $\mathbb{Z}^{4}$ to study the relation between these two flavours of well-composedness in higher dimensions. As depicted in Figure 2, we can define the digital subset of $\mathbb{Z}^{n}$ :

$$
\begin{aligned}
\mathcal{X}:=\{\{0,0,0,0\},\{0,0,0,1\},\{0,0,1,1\},\{0,1,1,1\}, \\
\\
\{1,1,1,1\},\{1,1,1,0\},\{1,1,0,0\},\{1,0,0,0\}\} .
\end{aligned}
$$

and its continuous analog (up to a translation) $X_{0}:=\varphi_{4}(\mathcal{X})$, where $\varphi_{d}$ is a bijection between $(\mathbb{Z} / 2)^{d}$ and $\mathcal{K}^{d}$ defined such that:

$$
\forall x \in \mathbb{Z} / 2, \quad \varphi(x):= \begin{cases}{[x, x+1]} & \text { when } x \in \mathbb{Z}, \\ \left\{x+\frac{1}{2}\right\} & \text { otherwise }\end{cases}
$$

and $\forall x \in(\mathbb{Z} / 2)^{d}, \varphi_{d}(x):=\times_{i=1}^{d} \varphi\left(x_{i}\right)$ where, as usual, $x_{i}$ denotes the $i^{\text {th }}$ coordinate of $x$. Let us check that $\mathcal{X}$ is DWC (see Figure 2). It is easy to observe that it does not contain any $2 \mathrm{D}$ critical configuration. Now, to observe that there is no primary or secondary 3D critical configuration, we can simply look at the 83 -faces (including the interior and the exterior cubes): since each one contains exactly 4 points of $\mathcal{X}$, they contain neither a primary critical configuration (made of 2 points) nor a secondary critical configuration (made of 6 points in the $3 \mathrm{D}$ case). Finally, we observe that the only $4 \mathrm{D}$ block that we have to consider is $\{0,1\}^{4}$ which contains 8 points of $\mathcal{X}$, and then 8 points of $\mathcal{X}^{c}$, and then $\mathcal{X}$ contains neither a primary nor a secondary $4 \mathrm{D}$ critical configuration.

Property 10. The digital set $\mathcal{X}$ is $D W C$.

\subsection{Finding a cubical set $\widetilde{X}\left(x^{0}\right)$ homotopy equivalent to $A$}

Let us start with the following proposition. 
Proposition 11. Let $X$ be a cubical set in $\mathbb{R}^{n}, x^{0}$ be a point of $X \cap \mathbb{Z}^{n}$, and $A:=X \backslash\left\{x^{0}\right\}$. Then, the set: $\widetilde{X}\left(x^{0}\right):=\left\{x \in A ;\left\|x-x^{0}\right\|_{\infty} \geq 1\right\}$, is cubical.

Proof. Our aim is to prove that $\widetilde{X}\left(x^{0}\right)$ is equal to $\cup\left\{h \in \mathcal{K}(X) ; x^{0} \notin h\right\}$. This way, we will be able to conclude that $\widetilde{X}\left(x^{0}\right)$ is equal to $\cup\{h \in \mathcal{K}(A)\}$ and then it is a cubical set (since it is made of cubes and closed under inclusion).

First, let us prove that we have: $\widetilde{X}\left(x^{0}\right) \subseteq \cup\left\{h \in \mathcal{K}(X) ; x^{0} \notin h\right\}$. Let $x \in A$ be a point such that $\left\|x-x^{0}\right\|_{\infty} \geq 1$. Then, there exists $i^{*} \in \llbracket 1, n \rrbracket$ such that $\left\|x-x^{0}\right\|_{\infty}=\left|x_{i^{*}}-x_{i^{*}}^{0}\right| \geq 1$. Then two cases are possible:

- $x_{i^{*}}>x_{i^{*}}^{0}$, then $x_{i^{*}} \geq x_{i^{*}}^{0}+1,(1)$

- $x_{i^{*}}<x_{i^{*}}^{0}$, then $x_{i^{*}} \leq x_{i^{*}}^{0}-1$. (2)

Since $x \in A \subset X$ where $X$ is a cubical set, there exists a smaller face $h^{*} \in \mathcal{K}(X)$ (in the inclusion sense) such that $x \in h^{*}:=\times_{i \in \llbracket 1, n \rrbracket}\left[\left\lfloor x_{i}\right\rfloor,\left\lceil x_{i}\right\rceil\right]$. Then, $x^{0} \in h^{*}$ iff for each $i \in \llbracket 1, n \rrbracket, x_{i}^{0} \in\left[\left\lfloor x_{i}\right\rfloor,\left\lceil x_{i}\right\rceil\right]$. However, since in case (1), $x_{i^{*}}^{0} \leq x_{i^{*}}-1<$ $\left\lfloor x_{i^{*}}\right\rfloor$, and in case $(2), x_{i^{*}}^{0} \geq x_{i^{*}}+1>\left\lceil x_{i^{*}}\right\rceil$, then $x^{0} \notin h^{*}$. Obviously, $h^{*} \in \mathcal{K}(X)$ : otherwise, all the cubes containing $h^{*}$ do not belong to $\mathcal{K}(X)$, and then $x \notin X$ and then $x \notin A$. This way, there exists a cube $h \in \mathcal{K}(X)$ such that $x^{0} \notin h$ and $x \in h$.

Second, let us prove that: $\widetilde{X}\left(x^{0}\right) \supseteq \cup\left\{h \in \mathcal{K}(X) ; x^{0} \notin h\right\}$. Let $p$ be an element of $\cup\left\{h \in \mathcal{K}(X) ; x^{0} \notin h\right\}$. In other words, $p \in h \in \mathcal{K}(X)$ and $x^{0} \notin h$. Since $p \in h$ and $x^{0} \notin h, p \neq x^{0}$, and $h \in \mathcal{K}(X)$, then $p \in X$, and then $p \in A$. Now, let us write $h=\times_{i \in \llbracket 1, n \rrbracket}\left[h_{i}^{\min }, h_{i}^{\max }\right]$, where $h^{\min }, h^{\max } \in \mathbb{Z}^{n}$. Since $x^{0} \notin h$, there exists $i^{*} \in \llbracket 1, n \rrbracket$ such that $x_{i^{*}}^{0} \notin\left[h_{i^{*}}^{\min }, h_{i^{*}}^{\max }\right]$. Furthermore, we have:

- either $x_{i^{*}}^{0} \leq h_{i^{*}}^{\min }-1,(A)$

- or $x_{i^{*}}^{0} \geq h_{i^{*}}^{\max }+1$. $(B)$

Since $p \in h$, for each $i \in \llbracket 1, n \rrbracket$, we have $p_{i} \in\left[h_{i}^{\min }, h_{i}^{\max }\right]$, and then $p_{i^{*}} \in$ $\left[h_{i^{*}}^{\min }, h_{i^{*}}^{\max }\right]$. Then, in case $(A), x_{i^{*}}^{0} \leq h_{i^{*}}^{\min }-1 \leq p_{i^{*}}-1$, which leads to $p_{i^{*}}-x_{i^{*}}^{0} \geq$ 1 , and in case $(B), x_{i^{*}}^{0} \geq h_{i^{*}}^{\max }+1 \geq p_{i^{*}}+1$, which leads to $x_{i^{*}}^{0}-p_{i^{*}} \geq 1$. In both cases, we obtain that $\left\|p-x^{0}\right\|_{\infty} \geq 1$.

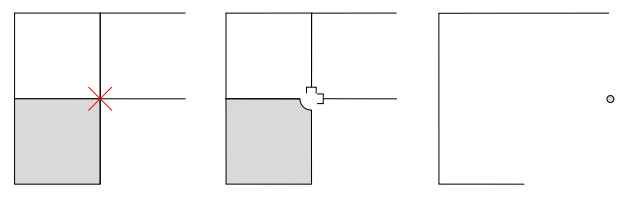

Fig. 3: $X \backslash\left\{x^{0}\right\}$ is homotopy equivalent to $\widetilde{X}\left(x^{0}\right)$ : From left to right, a cubical set $X$ (see the location of the central point $x^{0}$ in red), $X$ minus its central point $x^{0}$ and the new cubical set $\widetilde{X}\left(x^{0}\right)$ homotopy equivalent to $X \backslash\left\{x^{0}\right\}$.

Now, let us prove that $X \backslash\left\{x^{0}\right\}$ and $\widetilde{X}\left(x^{0}\right)$ are homotopy equivalent (as depicted on Figure 3). 
Proposition 12. Let $X$ be a cubical set in $\mathbb{R}^{n}, x^{0}$ be a point of $X \cap \mathbb{Z}^{n}$, and $A:=X \backslash\left\{x^{0}\right\}$. Then, $A$ is homotopy equivalent to $\widetilde{X}\left(x^{0}\right)$.

Proof. Let $f: A \rightarrow \mathbb{R}^{n}$ be the function defined such as:

$$
f(x):=\left\{\begin{array}{cl}
x & \text { when }\left\|x-x^{0}\right\|_{\infty} \geq 1, \\
x^{0}+\frac{x-x^{0}}{\left\|x-x^{0}\right\|_{\infty}} & \text { otherwise, }
\end{array}\right.
$$

and let $g: \widetilde{X}\left(x^{0}\right) \rightarrow A$ be the map from $\widetilde{X}\left(x^{0}\right)$ to $A$ such that:

$$
\forall x \in \tilde{X}\left(x^{0}\right), g(x)=x,
$$

which is possible since $\widetilde{X}\left(x^{0}\right) \subseteq A$. Now, let us proceed step by step.

Step 1: $A$ and $\widetilde{X}\left(x^{0}\right)$ are topological spaces. The sets $A$ and $\widetilde{X}\left(x^{0}\right)$ are topological spaces since they are subsets of $\mathbb{R}^{n}$ supplied with the usual Euclidian distance.

Step 2: $f$ is a map from $A$ to $\tilde{X}\left(x^{0}\right)$. Let $x$ be an element of $A$. When $\| x-$ $x^{0} \|_{\infty} \geq 1, f(x)=x$. This way, $f(x) \in A$ and $\left\|f(x)-x^{0}\right\|_{\infty} \geq 1$, then $f(x) \in$ $\widetilde{X}\left(x^{0}\right)$. When $\left\|x-x^{0}\right\|_{\infty}<1, f(x)=x^{0}+\frac{x-x^{0}}{\left\|x-x^{0}\right\|_{\infty}}$. This way, $\left\|f(x)-x^{0}\right\|_{\infty}=1$. Since $x \in A \subset X$ with $X$ a cubical set, there exists a cube $h \in \mathcal{K}(X)$ such that $x \in h$. Furthermore, this cube $h$ contains $x^{0}$ since all the cubes containing a point of $\left.\times_{i \in \llbracket 1, n \rrbracket}\right] x_{i}^{0}-1, x_{i}^{0}+1\left[\right.$ contain also $x^{0}$ (the cubes are defined relatively to integral coordinates). Since $h=\times_{i \in \llbracket 1, n \rrbracket} h_{i}$, then for each $i \in \llbracket 1, n \rrbracket, x_{i} \in h_{i}$, and

$$
(f(x))_{i}=x_{i}^{0}+\frac{x_{i}-x_{i}^{0}}{\left\|x-x^{0}\right\|_{\infty}} .
$$

Let us prove that this last equality shows that $f(x) \in h$. Since $n$ is finite, there exists some $i^{*} \in \llbracket 1, n \rrbracket$ such that: $\left\|x-x^{0}\right\|_{\infty}=\left|x_{i^{*}}-x_{i^{*}}^{0}\right|$, then:

$$
(f(x))_{i}=x_{i}^{0}+\frac{x_{i}-x_{i}^{0}}{\left|x_{i^{*}}-x_{i^{*}}^{0}\right|}
$$

Let us assume without constraint that $x_{i^{*}}>x_{i^{*}}^{0}$, then $(f(x))_{i^{*}}=x_{i^{*}}^{0}+1$. However, $x_{i^{*}}>x_{i^{*}}^{0}$ implies that $h_{i^{*}}=\left[x_{i^{*}}^{0}, x_{i^{*}}^{0}+1\right]$ since $h$ contains $x^{0}$. When $i \neq i^{*}$, since $x_{i} \in h_{i}$, and since $h \ni x^{0} \in \mathbb{Z}^{n}, h_{i}=\left[x_{i}^{0}, x_{i}^{0}+1\right]$ or $h_{i}=\left[x_{i}^{0}-1, x_{i}^{0}\right]$. Let us assume without constraint that $x_{i}>x_{i}^{0}$, then $h_{i}=\left[x_{i}^{0}, x_{i}^{0}+1\right]$. Because of $(P 1)$, it follows easily that $(f(x))_{i} \in h_{i}$ since $\frac{x_{i}-x_{i}^{0}}{\left|x_{i^{*}}-x_{i^{*}}^{0}\right|} \in[0,1]$.

Then, we have proven that when $\left\|x-x^{0}\right\|_{\infty}<1$, there exists $h \in \mathcal{K}(X)$ such that for any $i \in \llbracket 1, n \rrbracket,(f(x))_{i} \in h_{i}$, that is to say,

$$
f(x) \in h .
$$

Also, $x \neq x^{0}$, which is equivalent to $f(x) \neq x^{0}$. Then, $f(x) \in \widetilde{X}\left(x^{0}\right)$. 
Step 3: $g \circ f$ is homotopic to $\operatorname{Id}_{A}$.

- We can observe that $g \circ f: A \rightarrow A$ is the continuous function defined as:

$$
g \circ f=\left\{\begin{array}{cl}
x & \text { when }\left\|x-x^{0}\right\|_{\infty} \geq 1, \\
x^{0}+\frac{x-x^{0}}{\left\|x-x^{0}\right\|_{\infty}} & \text { otherwise. }
\end{array}\right.
$$

- Let $H: A \times[0,1] \rightarrow \mathbb{R}^{n}$ defined such that for any $x \in A$ and any $\lambda \in[0,1]$,

$$
H(x, \lambda):=\lambda x+(1-\lambda) g \circ f(x),
$$

then:

- $H$ is continuous as a composition of continuous functions,

- $H$ is from $A \times[0,1]$ to $A$ :

* when $\left\|x-x^{0}\right\|_{\infty} \geq 1, H(x, \lambda)=x \in A$,

* when $\left\|x-x^{0}\right\|_{\infty}<1, H(x, \lambda)=\lambda x+(1-\lambda) f(x)$. However, we have seen that in this case, cf. $(P 2)$, there exists a cube $h \in \mathcal{K}(X)$ such that $f(x) \in h$. Since $h$ is a cube, it is convex, and then $H(x, \lambda) \in h$. Then, $H(x, \lambda) \in X$. Also, we can prove that $H(x, \lambda) \neq x^{0}$ : the cases $\lambda=0$ and $\lambda=1$ are obvious; in the case $\lambda \in] 0,1[$, we can see that

$$
H(x, \lambda)=\lambda x+(1-\lambda)\left(x^{0}+\frac{x-x^{0}}{\left\|x-x^{0}\right\|_{\infty}}\right)
$$

and then, by assuming without constraints that $x^{0}=0$ and that for any $i \in \llbracket 1, n \rrbracket, x_{i} \geq 0$, we obtain that for any $i \in \llbracket 1, n \rrbracket$ :

$$
(H(x, \lambda))_{i}=\lambda x_{i}+(1-\lambda) \frac{x_{i}}{\|x\|_{\infty}}=\left(\lambda\left(\|x\|_{\infty}-1\right)+1\right) \frac{x_{i}}{\|x\|_{\infty}},
$$

then, because $\left(\|x\|_{\infty}-1\right)<1$ and $\frac{x_{i}}{\|x\|_{\infty}} \geq 0,(H(x, \lambda))_{i}$ is decreasing relatively to $\lambda$, and then

$$
x_{i} \leq(H(x, \lambda))_{i} \leq \frac{x_{i}}{\|x\|_{\infty}} .
$$

Since $x \neq 0$, there exists $i^{*}$ such that $x_{i^{*}} \neq 0$, and then such that $(H(x, \lambda))_{i^{*}}>0$ since $x_{i^{*}}>0$. Then, $H(x, \lambda) \neq 0$, that is, $H(x, \lambda) \neq$ $x^{0}$. Then, $H(x, \lambda) \in A$.

- We can see that $H(x, 0)=g \circ f(x), \forall x \in A$,

- We can also observe that $H(x, 1)=x, \forall x \in A$.

Then $g \circ f$ is homotopic to $\operatorname{Id}_{A}$.

Step 4: $f \circ g$ is homotopic to $\operatorname{Id}_{\widetilde{X}\left(x^{0}\right)}$. Since $f \circ g$ is equal to $\operatorname{Id}_{\widetilde{X}\left(x^{0}\right)}$, they are homotopic. 
Step 5: Conclusion. $A$ and $\widetilde{X}\left(x^{0}\right)$ are homotopically equivalent.

Corollary 13. Assuming the notations of Proposition 11, we can compute the homology groups of $A$ based on the ones of the cubical set $\widetilde{X}\left(x^{0}\right)$. Indeed, for each $i \in \mathbb{Z}$, we have the following equality: $\mathbb{H}_{i}(A)=\mathbb{H}_{i}\left(\widetilde{X}\left(x^{0}\right)\right)$.

Proof. This follows from Propositions 11 and 12.

\subsection{Choosing a particular point $x^{0} \in X$}

Let us begin with a simple property.

Property 14. The point $x^{0}:=(1,1,1,1)$ belongs to the boundary $X$ of $X_{0}=$ $\varphi_{4}(\mathcal{X})$

Proof. Let us recall that $\varphi_{4}(\mathcal{X})$ is a translation of the set $\mathrm{CA}(\mathcal{X})$ by a vector $v:=\left(\frac{1}{2}, \frac{1}{2}, \frac{1}{2}, \frac{1}{2}\right)$. Also, the point $p:=\left(\frac{1}{2}, \frac{1}{2}, \frac{1}{2}, \frac{1}{2}\right) \in \mathbb{R}^{4}$ belongs to $\mathrm{CA}(\mathcal{X})$ and does not belong to $\operatorname{Int}(\mathrm{CA}(\mathcal{X}))$ since there does not exist any topological open ball $B(p, \varepsilon), \varepsilon>0$, in $\mathbb{R}^{4}$ centered in $p$ and contained in $\operatorname{Int}(\mathrm{CA}(\mathcal{X}))$ since $B(p, \varepsilon)$ intersects $\operatorname{Int}\left(\mathrm{CA}\left(\mathcal{X}^{c}\right)\right)$. This way, $p$ belongs to $\operatorname{bdCA}(\mathcal{X})$. Finally, we obtain that the translation $x^{0}=p+v$ of $p$ by $v$ belongs to the translation $X$ of $\operatorname{bdCA}(\mathcal{X})$ by $v$.

\subsection{Computation of $\mathbb{H}(X, A)$}

Let us compute the relative homology groups $\mathbb{H}_{i}(X, A)$ for each $i \in \mathbb{Z}$. Obviously, since $\mathcal{C}_{k}(X, A)=0$ for $k \in \mathbb{Z} \backslash \llbracket 0,4 \rrbracket$, then $\mathbb{H}_{k}(X, A)=0$. Also, thanks to Proposition 4 , we know that $\mathbb{H}_{0}(X, A)=0$. To compute the other relative homology groups, we will use the exact homology sequence of $(X, A)$ discussed in Corollary 8: the sequence

$$
\cdots \rightarrow \mathbb{H}_{k+1}(A) \stackrel{\iota_{*}}{\longrightarrow} \mathbb{H}_{k+1}(X) \stackrel{\pi_{*}}{\longrightarrow} \mathbb{H}_{k+1}(X, A) \stackrel{\partial_{*}}{\longrightarrow} \mathbb{H}_{k}(A) \rightarrow \ldots
$$

is exact, and then by computing the homology groups $\mathbb{H}_{k}(X)$ and $\mathbb{H}_{k}(A)$ and using the first isomorphism theorem, we will be able to compute the local homology groups $\mathbb{H}_{k}(X, A)$ and to deduce if $X_{0}$ is a homology manifold or not.

Using CHomP [8], we compute the local homology groups $\mathbb{H}\left(\tilde{X}\left(x^{0}\right)\right)$ and $\mathbb{H}_{i}(X)$ for $i \in \llbracket 0,4 \rrbracket$, Using Corollary 13 we obtain $\mathbb{H}_{i}(A)$, and replacing this information in the long exact sequence discussed in Corollary 8, we obtain Figure 4. Let us compute $\mathbb{H}_{4}(X, A)$. By exactness, im $\pi_{4}=0=\operatorname{ker} \partial_{4}$ and $\mathbb{H}_{4}(X, A) /$ ker $\partial_{4} \simeq \operatorname{im} \partial_{4}=0$, then $\mathbb{H}_{4}(X, A)=0$. Now, let us compute $\mathbb{H}_{3}(X, A)$. By exactness, $\operatorname{im} \iota_{3}=0=\operatorname{ker} \pi_{3}$, and $\mathbb{Z} / \operatorname{ker} \pi_{3} \simeq \operatorname{im} \pi_{3} \simeq \mathbb{Z}$, then $\operatorname{im} \pi_{3}=$ ker $\partial_{3}=\mathbb{H}_{3}(X, A) \simeq \mathbb{Z}$. Concerning $\mathbb{H}_{2}(X, A)$, by exactness, im $\iota_{2}=0=$ $\operatorname{ker} \pi_{2}$, and $\mathbb{Z} / \operatorname{ker} \pi_{2} \simeq \operatorname{im} \pi_{2} \simeq \mathbb{Z} \simeq \operatorname{ker} \partial_{2}$. Also, $\operatorname{ker}\left(\iota_{1}\right)=\mathbb{Z}=\operatorname{im} \partial_{2}$ and $\mathbb{H}_{2}(X, A) / \operatorname{ker} \partial_{2} \simeq \operatorname{im} \partial_{2}$ imply that: $\mathbb{H}_{2}(X, A) \simeq \mathbb{Z}^{2}$. Finally, let us compute $\mathbb{H}_{1}(X, A)$. By exactness, im $\pi_{1}=0=\operatorname{ker} \partial_{1}$. Also, $\operatorname{ker} \pi_{0}=\mathbb{Z} \simeq \operatorname{im} \iota_{0}$, and $\mathbb{Z} / \operatorname{ker} \iota_{0} \simeq \operatorname{im} \iota_{0}$ imply that $\operatorname{ker} \iota_{0}=0=\operatorname{im} \partial_{1}$. Then, $\mathbb{H}_{1}(X, A)=0$. 


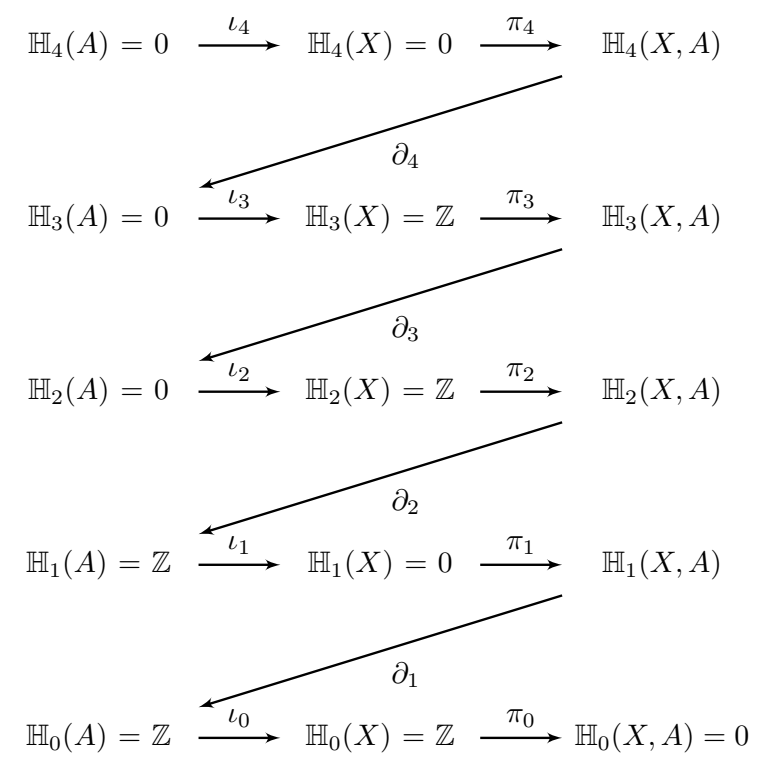

Fig. 4: Long exact sequence of the cubical pair $(X, A)$.

\subsection{Our final observation}

Since we have $\mathbb{H}_{2}(X, A) \simeq \mathbb{Z}^{2} \neq 0, X$ is not a homology 3-manifold, and then it is not a topological 3-manifold, which implies that DWCness does not imply CWCness in $n \mathrm{D}$, which contradicts the conjecture arguing that DWCness and CWCness are equivalent in $n \mathrm{D}$ on cubical grids [2]. Furthermore, this counterexample shows that a digital set which is well-composed in the sense of Alexandrov $(A W C)[17,6]$ is not always CWC, since it has been proven in [5] that AWCness and DWCness are equivalent in $n \mathrm{D}$.

\section{Conclusion}

We have shown in this paper that even if CWCness and DWCness are equivalent in $2 \mathrm{D}$ and in $3 \mathrm{D}$, it is not true in $4 \mathrm{D}$. As future works, we plan to study if CWCness implies DWCness in $n \mathrm{D}$.

\section{References}

1. Pavel S. Alexandrov. Combinatorial topology, volume 1-3. Graylock, 1956.

2. Nicolas Boutry. A study of well-composedness in $n-D$. $\mathrm{PhD}$ thesis, Université Paris-Est, France, 2016. 
3. Nicolas Boutry, Thierry Géraud, and Laurent Najman. How to make $n$-D functions digitally well-composed in a self-dual way. In J.A. Benediktsson, J. Chanussot, L. Najman, and H. Talbot, editors, Mathematical Morphology and Its Application to Signal and Image Processing - Proceedings of the 12th International Symposium on Mathematical Morphology (ISMM), volume 9082 of Lecture Notes in Computer Science, pages 561-572, Reykjavik, Iceland, 2015. Springer.

4. Nicolas Boutry, Thierry Géraud, and Laurent Najman. A tutorial on wellcomposedness. In Journal of Mathematical Imaging and Vision, 2017.

5. Nicolas Boutry, Laurent Najman, and Thierry Géraud. About the equivalence between AWCness and DWCness. Research report, LIGM - Laboratoire d'Informatique Gaspard-Monge ; LRDE - Laboratoire de Recherche et de Développement de l'EPITA, October 2016.

6. Nicolas Boutry, Laurent Najman, and Thierry Géraud. Well-composedness in Alexandrov spaces implies digital well-composedness in $\mathbb{Z}^{n}$. In Discrete Geometry for Computer Imagery, LNCS, page 225. Springer, 2017.

7. Vicent Caselles and Pascal Monasse. Geometric description of images as topographic maps, ser. Lecture Notes in Mathematics. Springer-Verlag, 1984, 2009.

8. CHomP. http://chomp.rutgers.edu/software/.

9. Thierry Géraud, Edwin Carlinet, Sébastien Crozet, and Laurent Najman. A quasilinear algorithm to compute the tree of shapes of nd images. In International Symposium on Mathematical Morphology and Its Applications to Signal and Image Processing, pages 98-110. Springer, 2013.

10. Tomasz Kaczynski, Konstantin Mischaikow, and Marian Mrozek. Computational homology, volume 157. Springer Science \& Business Media, 2006.

11. John L Kelley. General Topology, volume 27 of Graduate Texts in Mathematics. Springer, 1955.

12. Jacques-Olivier Lachaud. Espaces non-euclidiens et analyse d'image: modèles déformables riemanniens et discrets, topologie et géométrie discrète. $\mathrm{PhD}$ thesis, Université Sciences et Technologies-Bordeaux I, 2006.

13. Jacques-Olivier Lachaud and Boris Thibert. Properties of gauss digitized shapes and digital surface integration. Journal of Mathematical Imaging and Vision, $54(2): 162-180,2016$.

14. Longin Jan Latecki. 3d well-composed pictures. Graphical Models and Image Processing, 59(3):164-172, 1997.

15. Longin Jan Latecki. Well-composed sets. Advances in Imaging and Electron Physics, 112:95-163, 2000.

16. John Lee. Introduction to topological manifolds, volume 940. Springer Science \& Business Media, 2010.

17. Laurent Najman and Thierry Géraud. Discrete set-valued continuity and interpolation. In Proc. of the Intl. Conf. on Mathematical Morphology (ISMM), volume 7883 of $L N C S$, pages 37-48. Springer, 2013.

18. AA Ranicki, A Casson, D Sullivan, M Armstrong, C Rourke, and G Cooke. The hauptvermutung book. Collection of papers by Casson, Sullivan, Armstrong, Cooke, Rourke and Ranicki, K-Monographs in Mathematics, 1, 1996. 